\title{
Chapter 7 \\ Contributing to Restoration of Tidal Flats in Miyagi Prefecture's Moune Bay Following the Great East Japan Earthquake and Tsunami
}

\author{
Susumu Chiba, Takeshi Sonoda, Makoto Hatakeyama, \\ and Katsuhide Yokoyama
}

\begin{abstract}
The Great East Japan Earthquake destroyed many artificial structures on the coast of northeastern Japan, and as a result it restored wetlands in many places. To conserve these wetlands, we started to estimate the ecological and economic values of a restored mudflat in Moune Bay, Miyagi Prefecture, Japan. Our tasks could be classified into three main categories: (1) establish the distribution of commercially important clams; (2) investigate the dynamics of the benthic community inhabiting the tidal flats and the floor of the bay; and (3) create a system so that monitoring of the tidal flat environment-including the two tasks mentionedcould be performed by nonspecialists. We hope that our actions will help to stimulate discussion about how Japan's coastal areas should be used.
\end{abstract}

Keywords Asari • Moune Bay • Tidal flat • Tsunami • Wetland

\subsection{The Impact of the Great East Japan Earthquake and Tsunami on the Sanriku Coast}

The coast of the northern Tohoku region, stretching from the city of Ishinomaki in Miyagi Prefecture to the city of Hachinohe in Aomori Prefecture, is a "ria" coast, consisting of a collection of bays of varying sizes that back onto steep mountains.

\footnotetext{
S. Chiba $(\bowtie) \cdot$ T. Sonoda

Faculty of Bioindustry, Tokyo University of Agriculture, Abashiri, Hokkaido 099-2493, Japan e-mail: s2chiba@bioindustry.nodai.ac.jp

M. Hatakeyama

Mori wa Umi no Koibito (Non Profit Organization), Kesennuma, Miyagi 988-0527, Japan

K. Yokoyama

Department of Civil and Environmental Engineering, Tokyo Metropolitan University,

Hachioji, Tokyo 192-0397, Japan
} 
These inlets protected by mountains proved to be good harbors, and many small settlements formed on what flat land there was around the bays. However, it was these very topographic features of the rias that amplified the power of the massive tsunami on March 11, 2011, extending the damage right up to the high ground. The giant wave devastated most settlements on the Sanriku coast, claiming the lives of many residents. Furthermore, once the tsunami had subsided it became apparent that the ground had sunk by tens of centimeters. This subsidence was enough to further shrink the already scarce area of flat land available in the Sanriku bays, and some areas where people had resided were now part of the sea.

Even two and a half years later, the pace of recovery is slow and little progress has been made. Many Sanriku coast residents affected by the disaster still live in temporary housing, partly because many cannot return to their ruined homes, but also reflecting the residents' uncertainty over whether it is wise to return to the area. Amidst such uncertainty, the local authorities made a unilateral decision to build seawalls 10 to $15 \mathrm{~m}$ high around much of the Sanriku coast. These giant walls might perhaps give the residents some peace of mind, but at the same time will deprive them of the view from the land and the breeze from the wind that once swept across the sea. The fishermen will likely have to scale these walls each day to check the sea conditions. And will the tourists who visit the Sanriku region for its beautiful views still leave satisfied? Although nothing is more important for a society than protecting life, the plan to obscure the Sanriku coast with seawalls leaves plenty of scope for debate.

\subsection{Moune Bay and the NPO Mori wa Umi no Koibito}

Moune Bay is a small inlet typical of those in the Sanriku region, located east of the city of Kesennuma in Miyagi Prefecture (Fig. 7.1). The bay settlement was unavoidably engulfed by the massive tsunami, and the land also subsided $70 \mathrm{~cm}$, turning much of the area into tidal flats, or wetlands filled with halophytic plants. One can hardly begin to imagine the sense of loss and despair felt by the residents who lost their home, a place to which to return.

In the midst of the post-tsunami chaos, the NPO Mori wa Umi no Koibito (translated literally as "the forest is longing for the sea") has led discussion on land usage in the Moune district, with a view to restoring the tidal flats. This NPO is led by Shigeatsu Hatakeyama, a fisheries operator who was among the world's first proponents of tree-planting initiatives to protect the ocean. Hatakeyama's book Mori wa umi no koibito (published by Bungeishunju Ltd.) is well-known in Japan. Thanks to his book and activities, the Moune district had been a focus of attention even before the disaster, hosting numerous visitors from both within Japan and overseas, and many of its residents were highly conscious of the relationship between humans and nature. Even with this background, however, it was only natural that after the tsunami the residents were torn between reclaiming as much of the former land as they could or starting completely afresh. It was not a decision to be taken lightly. Eventually, with residents in two minds, the deciding factor proved to be the discovery of tiny asari (Japanese littleneck) clams, measuring less than $5 \mathrm{~mm}$, on what had previously been a road. 


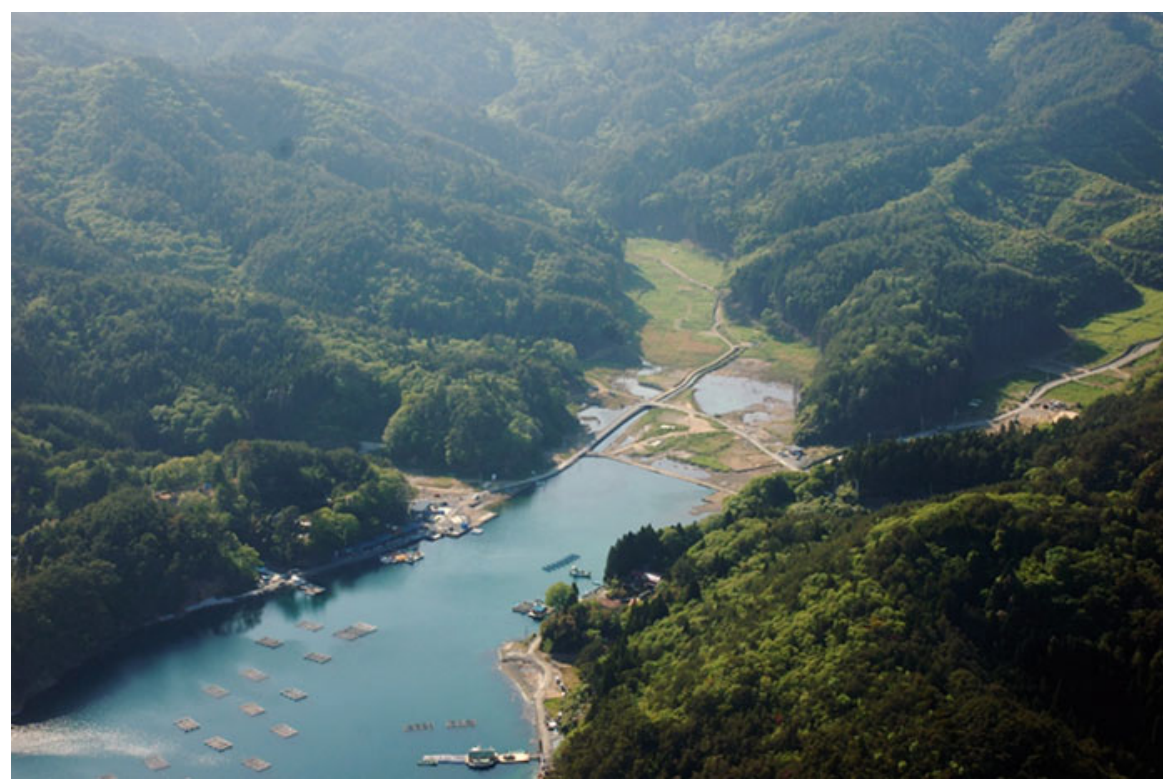

Fig. 7.1 Moune Bay. The bay settlement was engulfed by the tsunami and the land subsided. (Photograph courtesy of IDEA Consultants, Inc.)

The asari clam is a bivalve that has been used for food in Japan since ancient times. In recent years, however, the quantity caught across the country has plunged. Although there are several reasons for this drastic decline in asari numbers, the shift in the way humans have used coastal areas in recent years is a major factor. The human desire to utilize land to its limits, combined with a rapid advance in civil engineering technology, has seen solid land extended to wetland and shore areas, replacing the ecotone - the vague boundary that separates land from sea—with a clear-cut demarcation. This demarcation has made it possible to expand the territory available for human use, providing additional habitable area even in the Sanriku region, where flat land is scarce. However, it was in this vague boundary between land and sea that natural resources were most abundant. The asari clam was one of these.

In recent years, the Japanese coastline has paid the price for this short-sighted pursuit of profit, with many of the natural assets taken for granted in the past quietly starting to disappear. The Great East Japan Earthquake was a terrible disaster that caused unspeakable loss-that fact is beyond debate. However, it is also an unfortunate truth that the resulting breakdown of the boundary between land and sea allowed us to once again catch a glimpse of nature's fading bounty. It was in fact not until the 1960s that the coast of Moune Bay was filled in to its pre-earthquake form; as recently as 50 years ago the area had originally been wetland, consisting mainly of tidal flats. So perhaps the discovery of asari clams on a road that the earthquake had turned into a tidal flat made Moune residents nostalgic for the old days, or perhaps it gave them new hope for the future. Either way, the discovery of the clams proved the impetus for a petition presented to Kesennuma's city authority in June 
2012, stating the residents' intent to live in harmony with the ocean, without relying on seawalls. At the time, this was the only such decision made by residents on the Sanriku coast.

\subsection{The Role of the Tokyo University of Agriculture Team}

The discovery of asari clams in the area gave the Tokyo University of Agriculture group an opportunity to cooperate in the restoration of tidal flats in the Moune bay. Our tasks could be classified into three main categories: (1) establish the distribution of asari clams within the Moune area; (2) investigate the organisms inhabiting the tidal flats and the floor of the bay beyond, and how they changed over time (in other words, study the dynamics of the benthic community); and (3) create a system so that monitoring of the tidal flat environment-including the two tasks abovecould be performed by nonspecialists. Of these three tasks, our responsibility for the third was particularly large.

As part of its environmental education program, the NPO Mori wa Umi no Koibito holds courses attended by many local elementary and junior high school students. If we could work with the NPO to develop our initiatives into a monitoringbased education program for children, it would enable monitoring to be performed on an ongoing basis. And it would be a wonderful added bonus if the experience of monitoring as children resulted in an increased number of adults with an appreciation of all that they owed to their natural surroundings.

\subsection{Asari Clams}

We selected a survey site in the back of the Nishi-moune bay, consisting of a $3,000 \mathrm{~m}^{2}$ area enclosed by two small rivers, the Nishi-moune River and the Higashimoune River (Fig. 7.2). The area previously contained a concrete-covered coast
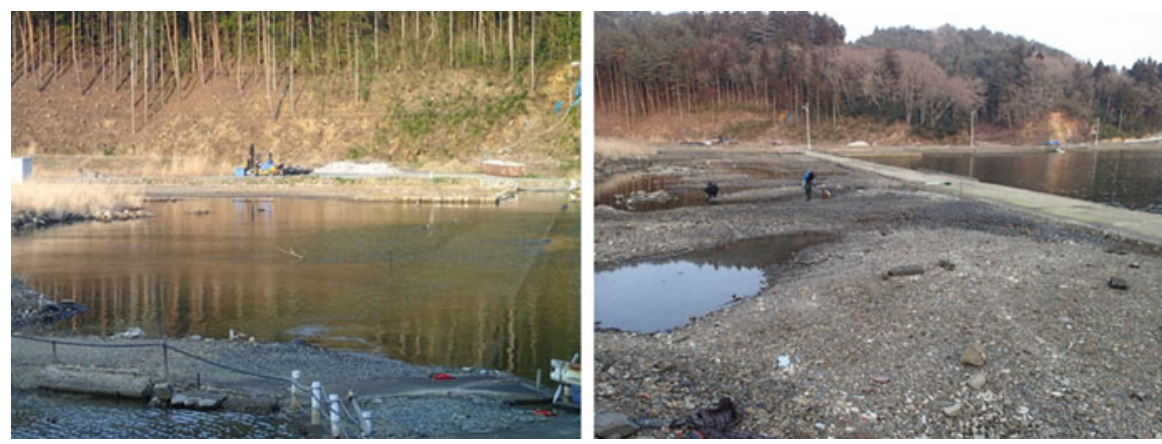

Fig. 7.2 A monitoring area of tidal flats at high tide (left) and low tide (right) 
Fig. 7.3 Asari clams and other benthos collected from quadrat of $20 \times 20 \mathrm{~cm}$

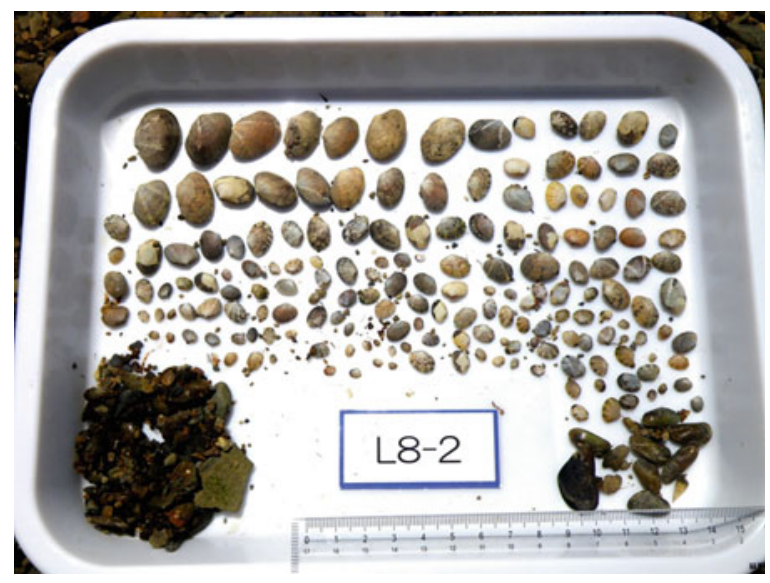

wall that served as both an embankment and a road. Located behind this had been wind-blocking trees, houses, and farmland, but the 70-cm ground subsidence means that the area is now completely submerged at high tide. We have visited the site once every 6 weeks since the study began in May 2012, continuing the same survey work.

From our investigation results it became evident that the asari clams discovered on the tidal flat that had formerly been land were not a chance finding: they were clearly inhabiting the area (Fig. 7.3). Most of the clams collected in 2012 were still small, measuring less than $10 \mathrm{~mm}$ in length. As an overall estimate taking into consideration factors such as the frequency of shell length and the number and width of ring patterns on shells, the clams measuring less than $10 \mathrm{~mm}$ were believed to be almost exactly 1 year old. This finding suggests that the spat (young shellfish) emerged after the March 2011 earthquake and subsequently inhabited the tidal flat.

The concentration of juveniles of the asari clams exceeded 6,500 individuals per square meter in highly populated areas, and exceeded 4,000 individuals on average in the tidal flat that had formed beside the former embankment road. Most surprisingly, this concentration was as high as any of the major asari clam production sites in Japan. At present, however, this outstanding level of production is limited to one part of the tidal flat, and it is an environment that, having once been dry land, is not now ideally suited to either humans or marine life. If anything, we should be impressed by the fact that the area was populated with so many clams despite the poor environment. Indeed, the discovery of the clams is of major significance in considering the value of reviving the Moune tidal flats.

\subsection{Other Benthos}

Asari clams are a symbol of the revival of the Moune tidal flats, and an important species from the fishing industry's perspective because of their market value. However, from an ecological perspective asari clams are merely one species in the 
tidal flat ecosystem. As the ecosystem comprises a combination of mutual interactions between various organisms and the environment, it is impossible to gain an understanding of it from studying a single species in isolation. An essential research task, therefore, is to study the dynamics of other benthic communities, even if the ultimate objective remains asari clam cultivation.

In addition to asari clams, the existence of 16 species of shellfish, 4 species of decapod crustacean such as shrimps and crabs, and 3 species of polychaetes such as ragworms has been confirmed at present. When we began the study, most of these organisms were significantly smaller than they would be when fully grown. Similar to the asari clams, they are therefore believed to have entered the newly formed tidal flat at a later date, rather than being washed inland by the tsunami. With each subsequent study, as time passed following the disaster, the number of species present gradually increased, suggesting that the tidal flat is steadily becoming closer to functioning normally again.

\subsection{Development of Monitoring Techniques}

We are currently working to develop monitoring techniques in the hope that local residents-particularly elementary and junior high school students-will eventually take over the task of monitoring the tidal flats (Fig. 7.4). Above all these

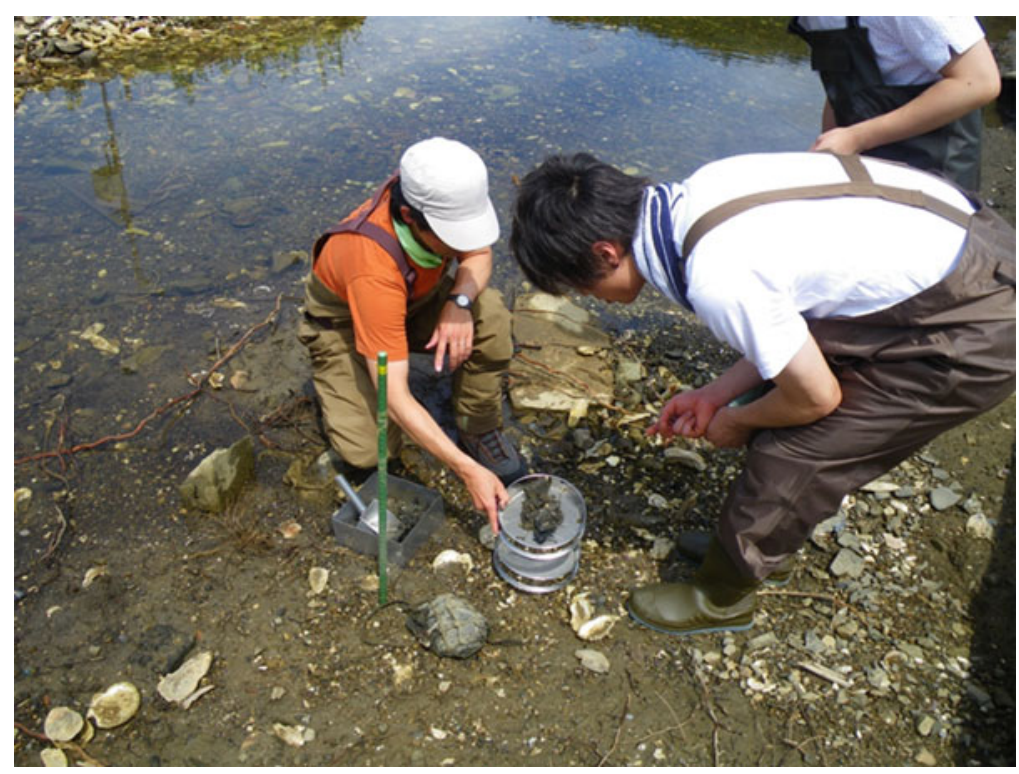

Fig. 7.4 Development of accurate and simple monitoring techniques for local residents 
techniques need to be simple, so that anyone can perform them. At the same time, however, we are aiming to collect data that will actually be useful 10 or 50 years in the future.

The most difficult task for monitors is classification of the benthos. With the exception of asari clams, nonspecialists are unable to identify by name many of the benthic species. Although the difference between shrimps, crabs, and shellfish is understood, at levels above this many organisms appear similar to the untrained eye. To address this issue, we began by taking photographs of each benthic organism collected and are currently working to create illustrations annotated with their characteristics.

The biggest challenge during this task is likely to be capturing children's interest: if they do not find the monitoring process interesting, they will not keep it up. As it is important that we find the facts we need, we are reviewing the monitoring methods through trial and error so that we do not end up with an ineffectual plan created from an adult perspective.

\subsection{Future Challenges and Actions}

Restoration of the Moune wetlands has only just begun, and it is likely that we will be forced to constantly review our plans in response to a range of unpredictable political and economic factors. The issue of how to use the wetlands created by the earthquake and tsunami can only be decided by the local residents themselves. However, nature, including wetlands, offers much of value that is essential for human life. We believe it is science's role and responsibility to society to explain this value in a clear, straightforward manner. In Japan there is a tendency to classify things as "useful" or "not useful" according to the direct benefit they deliver to humans. This mindset means that, although people realize that environmental conservation is a positive and important thing, it is often difficult to gain support for it.

We believe that, rather than complaining, it is best to begin by accepting the situation as it is. We also believe that the first step toward restoration of the wetlands is identifying and communicating what the tidal flats offer that is of value, focusing on the asari clams that served as the impetus for restoration efforts. We hope that this first step will help to stimulate discussion about how Japan's coastal areas should be used.

Open Access This chapter is distributed under the terms of the Creative Commons Attribution Noncommercial License, which permits any noncommercial use, distribution, and reproduction in any medium, provided the original author(s) and source are credited. 